\title{
Modification of Spatial Distribution in Primitive and Point Configuration Texture Model
}

\author{
Yasushi Kobayashi and Akira Asano \\ Hiroshima University, Higashi-Hiroshima, Hiroshima 739-8521 JAPAN, \\ sunweb@hiroshima-u.ac.jp
}

\begin{abstract}
We have investigated "Primitive and Point Configuration (PPC) texture model," which regards a texture to be composed by arranging grains that are derived from one or a few primitives by some modification. The PPC texture model is able to characterize primitive shapes and grain location configuration independently. We propose in this paper methods of manipulating for the grain location configuration. These methods enable to manipulate global characteristics of a texture independently on local ones. Our methods modify the grain configuration using random point process and generate different textures while preserving the original primitive shape.
\end{abstract}

\section{Introduction}

Texture classification and discrimination are main topics of image analysis. Various methods of texture analysis, for example the cooccurence matrix method and the spatial frequency method, have been proposed [1]. Texture characteristics measurements, which are main aims of the above popular methods, are often employed for texture classification and discrimination. We present a framework of texture analysis from another point of view in this paper. We have proposed a method of describing how a texture is generated under a texture model. Our proposed model, called "Primitive and Point Configuration (PPC) texture model," assumes that a texture is an arrangement of grains that are derived from one or a few primitives [2]. We have also proposed an estimation method of the primitive shape and grain location configuration in case that the grains are derived from one primitive by homothetic magnification $[2,6,7]$.

The PPC texture model is able to characterize a primitive, which express local characteristics, and grain location configuration, which express global characteristics, independently. Using this advantage, we propose in this paper several methods of deriving a different texture by modifying the global characteristics of the original ones while preserving the local ones. The grain location configuration is derived by morphological skeleton $[3,4]$ using the primitive as the structuring element, if we assume that grains are derived by homothetic magnification of one primitive and that the primitive shape has been already estimated. The morphological skeleton is explained as follows: Suppose covering the object with preferably large homothetic magnifications of a structuring element in the following way. At first we locate the largest magnification contained within the object. 
Then gradually smaller magnifications are employed for covering the residual region until the whole object is covered. The skeleton is defined as the union of the origins of all the employed magnifications of the structuring element. If the skeleton of a texture is calculated with the primitive as the structuring element, the skeleton is the set of points where magnifications of the primitive are located to reconstruct the original texture. Thus the skeleton of the target texture is regarded as the grain location configuration.

In this paper, we modify the grain location configuration of a texture and regenerate the point configuration. We regard the grain location configuration to be generated by a spatial random point process, and newly generate a grain location configuration by modifying the spatial point process. The modification of the grain location configuration is applicable, for example, to the conversion of random textures to regular ones with the same periodicity for comparison, and the generation of similar textures by parameterizing the random point process of grain location configuration. We develop several methods of modifying the grain location configuration while preserving the primitive in this paper. These methods can manipulate global impression of the target texture while preserving local characteristics. Our developed methods are thinning with restrictions, regeneration of a point configuration with preserving characteristics of a texture, and modification based on the correlation between sizes.

\section{Morphological skeleton}

We employ the ordinary morphological skeleton in this model. Let $X$ be a binary image set, and $B$ be a structuring element. The skeleton $S K(X, B)$ is defined as follows:

$$
\begin{aligned}
S K(X, B) & =\bigcup_{n=0}^{\infty} S K_{n}(X, B), \\
S K_{n}(X, B) & =(X \ominus n \check{B})-(X \ominus n \check{B})_{B},
\end{aligned}
$$

where $\ominus$ denotes Minkowski set substraction, $\check{B}$ denotes the symmetrical set of $B$ with respect to the origin, and $n B$ is the $n$-times homothetic magnification of $B$ defined as follows:

$$
\begin{aligned}
n B & =B \oplus C \oplus \ldots \oplus C \quad((n-1)-\text { times of } \oplus) \\
0 B & =0
\end{aligned}
$$

where $C$ is another small structuring element. This definition is different from the usual one, however, we employ this definition to avoid the inconvenience that the difference between $n B$ and $(n+1) B$ is too large for large $B$ in the original definition. We set $C$ to $2 \times 2$-pixel square in our experiment. $X_{B}$ denotes the opening of $X$ by $B$, defined as follows:

$$
X_{B}=(X \ominus \check{B}) \oplus B,
$$


where $\oplus$ denotes Minkowski set addition. The grayscale image composed by assigning a pixel value $n$ to the pixels in $S K_{n}(X, B)$ is referred as the medial axis transform. The original binary image is reconstructed by locating $n B$ on every pixel of $S K_{n}(X, B)$ and calculating the union for all $n$. This indicates that $S K_{n}(X, B)$ is regarded as the grain location configuration of the texture $X$ if we assume that $B$ is the estimated primitive and $n B$ 's are the grains.
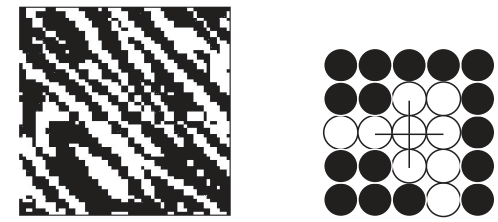

Fig. 1. (a) An example texture. (b) An estimation of the primitive.
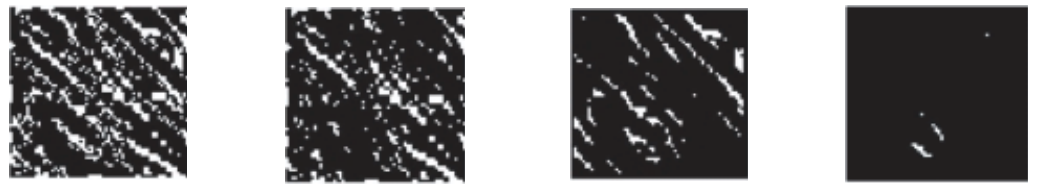

Fig. 2. (a) The skeleton of Fig.1(a). (b) $S K_{1}$, (c) $S K_{2}$, and (d) $S K_{3}$.

\section{Modification of grain location configuration}

Figure 1 shows an example binary texture $[8,9]$ and an estimation of its primitive. When the skeleton is obtained using a primitive estimated in a certain way, the white pixels showing the grain location configuration appear in the skeleton image. We regard in this paper the grain location configuration to be generated by a spatial point process with a probability distribution. Since the grain size to be located is assigned to each of the while pixels in the skeleton image, the white pixels are regarded as the point configuration, denoted $\phi$, generated by a marked point process $\Phi$. As shown in Fig. 2, a skeleton image is separable into $S K_{n}(X, B)$ each of which contains the pixels corresponding to only one size. Since the point configuration on $S K_{n}$, denoted $\phi_{n}$, is not assigned any size, it 
is regarded to be generated by a simple point process $\Phi_{n}$. A modified texture is generated by a modification of $\Phi_{n}$. We restrict ourselves to the manipulation of local point density and local intersize correlations, which evaluate relationships between the assigned sizes of neighboring pixels.

\section{1 thinning}

Thinning is an elimination of a part of point configuration by a given point process. In the following modifications, we carry out the thinning while preserving characteristics of images as much as possible.

elimination of isolated points: Since isolated points, which are not adjacent to any other points in $\phi_{n}$, have small extents in the original image, they are considered noise and the influence of its elimination to the image is small. We modified the skeleton by eliminating isolated points in $\phi_{1}$ only. The image regenerated from the modified skeletons is shown in Fig. 3(a). The comparison of Fig. 1(a) and Fig. 3(a), indicates that the characteristics of the image are almost preserved, although isolated points are eliminated from the original image.

elimination of concentrated points: If isolated points for large $n$ 's are eliminated, characteristics of the original image are spoiled since the grain located as such point is significant. We define here a "concentrated point" as a white pixel surrounded by white pixels in all of 4-neighborhood. Since one grain at one of concentrated points is not so significant in the original texture, elimination of such points is effective for thinning with preserving characteristics of $\phi_{n}$ for large $n$. We carried out a modification by eliminating concentrated points and preserving the other points in $\phi_{2}$ and $\phi_{3}$. The image regenerated from the modified skeletons is shown in Fig. 3(b). According to the comparison of Fig. 1(a) and Fig. 3(b), characteristics of the original image are preserved by the elimination although a part of concentrated points are removed.
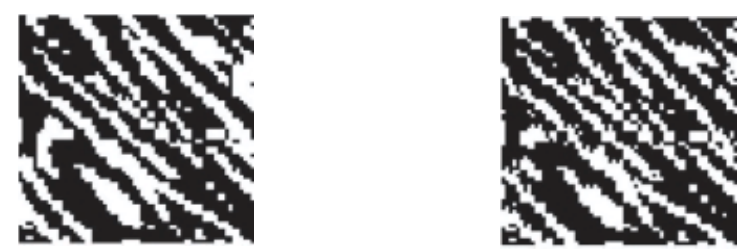

Fig. 3. (a) An example of thinning by elimination of the isolated points. (b) An example of thinning by elimination of the concentrated points. 


\subsection{Regeneration of point configuration}

In this subsection, we generate a different point configuration under certain restrictions on the number of points in a part of an image, and we observe how different the regenerated images are from the original ones.

Modification of point configuration based on the total number of points in a segment: We modify randomly the point configuration in each segment obtained by partitioning the original texture while preserving the total number of points in the segment, and we observe how the modified images are modified.

Suppose that the original point configurations $\phi_{n}(n=1,2,3)$ are partitioned into $5 \times 5$-pixel segments, and the point configuration in each segment is modified. A new point configuration $\psi_{n}$ that has the same total number of points as the original image in each segment is newly generated by randomly choosing the pixels in each segment by a uniform probability. Since points of $\psi_{n}$ are uniformly independently distributed in each segment, the characteristics of the original image in each segment are not preserved although global characteristics over the whole image are preserved. Figure 4(a) shows an example of the regenerated image.

Modification of point configuration where the total number of points in a segment is considered as the expectation of a probabilistic process: Suppose the $5 \times 5$-pixel segments similarly to the above, although the number of points in each segment is considered the expectation of a newly introduced random process. A Poisson point configuration $\psi_{n}^{\prime}$, whose expectation is the total number of points in each segment, is newly generated. In the example shown in Fig. 4(b), the differences between the total number of points in $\phi_{n}$ and $\psi_{n}^{\prime}$ were as follows:

$-n=1: \phi_{1}=678: \psi_{1}^{\prime}=651$,

$-n=2: \phi_{2}=207: \psi_{2}^{\prime}=201$,

$-n=3: \phi_{3}=13: \psi_{3}^{\prime}=9$.

In the original point configuration in each segment, there exists a correlation among the points. Since the points by Poisson point configuration are independently generated, the replacement of the original point configuration with Poisson point configuration removes any correlation preserving the density of points in each segment. It is shown in Fig. 4(b) that points are more scattered in each segment than the original, although the global distribution of white pixels in the original image is preserved.

\subsection{Modification based on intersize correlation}

It is observed in the grain location configuration that a point corresponding to a large-size grain is often surrounded by points corresponding to smaller-size 

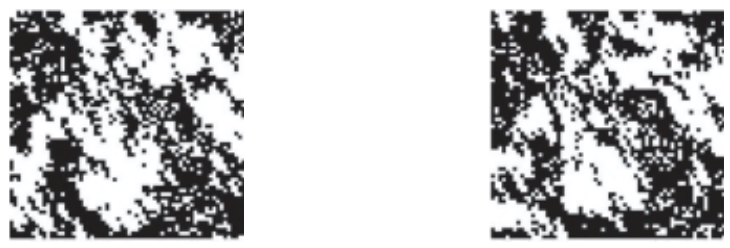

Fig. 4. (a) An example image regenerated based on the total number of points. (b) An example image regenerated based on the expectation.
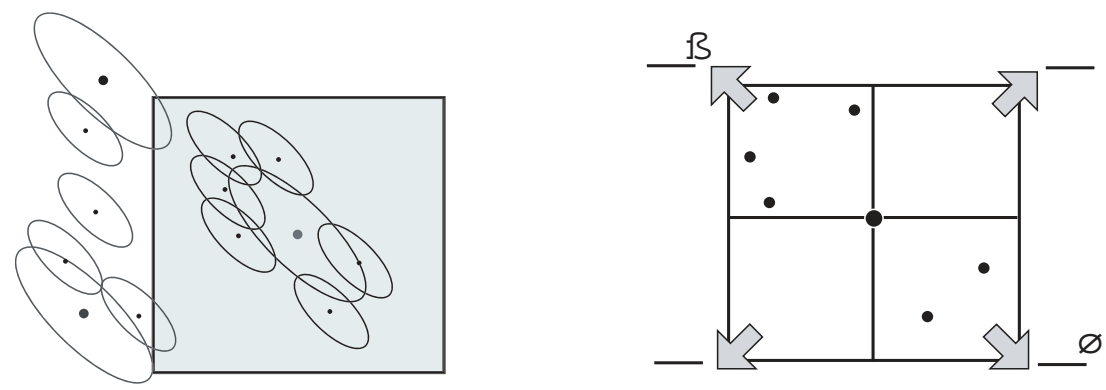

Fig. 5. (a) An example of grain location. Each ellipse indicates a grain. (b) Observed intersize correlation in the gray window in (a). 
grains. This indicates that there exists a correlation between the location of the point of size $n$ and that of size $n-1$. We call it intersize correlation. We consider here relocation of points while preserving the intersize correlation of the original texture.

We measure the intersize correlation in the original texture as follows: We assume a $11 \times 11$-pixel window centered at each point in $\phi_{n}$ for the largest $n$, as shown in Fig. 5(a). The window is divided into four subwindows as shown in Fig. 5(b), and the number of points in $S K_{n-1}$ in each subwindow is counted. The ratio of the count in each subwindow to the total number of points in the window in $S K_{n-1}$ is regarded as an appearance probability of the pixels of size $n-1$ arround the pixel of size $n$.

We generate a new texture by locating the points of size $n$, generating randomly the points of size $n-1$ arround the points of size $n$ following to the appearance probabilities estimated as the above from one of the points in $\phi_{n}$ for each of newly generated points of size $n-1$, and assigning corresponding grains to these point. Figure 6(a) shows an example of generated texture in case that the points of size $n=3$ are located randomly, while Fig. 6(b) shows the texture in case that these points are located regularly. These examples show that the approach using the intersize correlation succeeds manipulation of the global grain location configuration while preserving the local one.
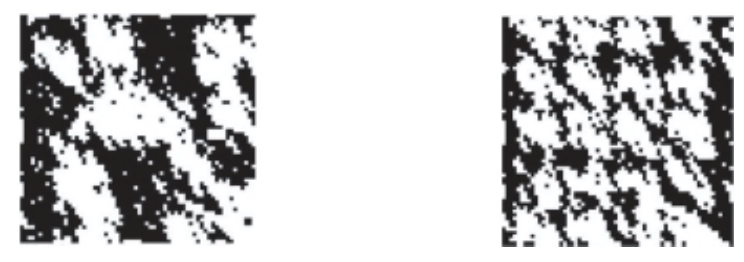

Fig. 6. (a) A regenerated image where the largest-size points located randomly, (b) a regenerated image where the largest-size points located regularly.

\section{Conclusions}

In this paper, we have proposed a texture modification method that modifies global characteristics of a texture while preserving local characteristics, based on the Primitive and Point Configuration (PPC) texture model. We have shown the experimental results of thinning, random regeneration of the grain location configuration, and modification based on the intersize correlation \}Although we have proposed the methods only for binary textures in this paper, we are investigating its extension to grayscale textures now. 


\section{References}

1. T. Ojala and M. Pietikäinen, Texture classification, in R. B. Fisher, ed., CVonline:The Evolving, Distributed, Non-Proprientry, On-Line Compendium of Computer Vision. (http://www.dai.ed.ac.uk/CVonline/LOCAL_COPIES/OJALA1/texclas.htm)

2. Asano, A., Ohkubo, T., Muneyasu, M., and Hinamoto, T.: Primitive and Point Configuration (PPC) Texture Model and Parameter Estimation using Mathematical Morphology, to be accepted in SCIA2003

3. Heijmans, H. J. A. M.:Morphological Image Operators. Academic Press (1994)

4. Maragos, P.:Pattern Spectrum and Multiscale Shape Representation. IEEE Trans. Pattern Anal. Machine Intell. 11 (1989) 701-706

5. Asano, A., Miyagawa, M., and Fujio, M.: Morphological texture analysis using optimization of structuring elements. Geometry, Morphology, and Computational Imaging, Springer LNCS 2616, (2003) 141-152

6. Asano, A., Ohkubo, T., Muneyasu, M., and Hinamoto, T.: Texture Primitive Description Using Morphological Skeleton. Proc. International Symposium on Mathematical Morphology VI (2002) 101-108

7. Asano, A., Miyagawa, M., and Fujio, M.: Texture Modelling by Optimal Gray Scale Structuring Elements using Morphological Pattern Spectrum. Proc. 15th International Conference on Pattern Recognition 3 (2000) 479-482

8. Brodatz, P.:Textures - A Photographic Album for Artists and Designer. Dover, New York (1966)

9. Randen, T.:Brodatz Textures, http://www.ux.his.no/ ${ }^{\sim}$ tranden/brodatz.html . 